\title{
L'usine hydroélectrique de Vogelgrün
}

\section{Vogelgrün hydro-electric power station}

\author{
M. LOUBATON
}

DIRECTEUR-ADJOINT

DE LA REGION D'ÉQUIPEMENT HYDRAULIQUE NORD

D'ÉLECTRICITÉ WDE FRANCE
PAR

ET

INGÉNIEUT A LA RÉGION D'ÉOUIPEMENT HKDRAULIOUE NOH D'ÉLECTHICITÉ DE FRANCE
L'usine hydro-électrique de Vogelgrün, dont la puissance installée sera de $156000 \mathrm{kVA}$ et la praduction annuelle, moyenne de 720 millions de $k W h$ en situation definitive, comprend quatre groupes et quatre déchurgeurs qui seront mis en service dans le courant de 1959.

Les turbines sont du type Kaplan, les alternateurs du type parapluie. Les trois pertuis de l'entrée de chaque groupe sont obturés chacun par ane vanne d̀ manouvre automatique.

Le schéma électrique de la centrale est du type bloc : alternatenr et transformateur étant relies sans interposition de disjoncteur.

L'usine a été fondée à l'intérieur d'une cuvette. étanche. Cette cuvette a été réalisée par injections d'argile et de ciment dans les alluvions. Dans la construction l'emploi de ciments d fort pourcentage de laitier a été généralisé et la proportion d'aciers durs arénelés augmentée.

\begin{abstract}
The Vogelgrïn hydro.electric power station, which will ultimately have an installed power output of $156000 \mathrm{kVA}$ and an annual output of $720000000 \mathrm{kWh}$, is equipped with four units and the necessary by-passing devices, which are expected to be put into service some time in 1959 .

The turbines are Kaplan units, with the alternators mounted above the turbines. The three intake sluices for each unit are each fitted with an automatic gate.

The electrical circuit in the power station is of the block type in which the alternator and transformer are directly connected together with a circnit breaker between them.

The power station is built in a sealed basin formed by grouting clay and cement into the alluvial gronnd. Cement with a high slag content was generally used in the construction work, as well as a large amount of irregular surface hard steel than is usually the case.
\end{abstract}

- une usine hydro-électrique;

- un canal de fuite de $1500 \mathrm{~m}$ de longueur (comportant un quai pour la Chambre de Commerce de Colmar en prolongement de l'écluse de raccordement du canal du Rhônc au Rhin).

Nous nous limiterons dans cet article à l'étude de l'Usine, dont nous traiterons des chapitres suivants :

-..- hydrologie et production,

-- description des ouvages,

- conduite des travaux. 


\section{I. - HYDROLOGIE ET PRODUCTION}

La chute de Vogelgrun étant située sur le Grand Canal d'Alsace et réalisée conformément au projet de 1925, son débit d'équipement est limité à celui dérivé à l'origine du canal a Kembs. Le bassin versant intéressé a pour surface $35930 \mathrm{~km}^{2}$ dont $1000 \mathrm{~km}^{2}$ de lacs et $700 \mathrm{~km}^{2}$ de glaciers, le module est de $29,51 / \mathrm{s}$, le débit du canal de $1080 \mathrm{~m}^{3} / \mathrm{s}$ et le cœfficient d'utilisation du canal par rapport au Rhin de 0,84 environ. Lacs et glaciers régularisent le régime du fleuve de manière fort appréciable puisque, pour le débit moyen de $1060 \mathrm{~m}^{3} / \mathrm{s}$ à Bâle (calculé sur 55 années), le débit moyen d'hiver se situe à $800 \mathrm{~m}^{3} / \mathrm{s}$ et celui d'été à $1300 \mathrm{~m}^{3} / \mathrm{s}$; le débit d'étiage dans les années sèches ne descend pas au-dessous de $400 \mathrm{~m} 3 / \mathrm{s}$ pendant 10 jours par an. Les crues annuelles normales du fleuve oscillent autour de $3000 \mathrm{~m}^{3} / \mathrm{s}$, la crue exceptionnelle de $5500 \mathrm{~m}^{3} / \mathrm{s}$ ne s'étant pas reproduite depuis 1876 .

A noter que le retour au lit du Rhin, pour les aménagements qui seront réalisés à l'aval de Vogelgrun, permettra d'accroitre le débit d'équipement en le portant de $1080 \mathrm{~m}^{3} / \mathrm{s}$ à $1400 \mathrm{~m}^{3} / \mathrm{s}$, débit qui sera atteint en moyenne 90 jours par an.

La hateur de chute variera entre $11,60 \mathrm{~m}$ et $15,50 \mathrm{~m}$ en fonction du niveau du Rhin à la restitution. Les valeurs moyennes de cette hauteur en situation provisoire el en situation définitive, c'est-à-dire avant et après exécution du barrage de Marckolsheim, seront respectivement de $13,40 \mathrm{~m}$ et $11,80 \mathrm{~m}$ environ.

La puissance installée sera de $140000 \mathrm{~kW}$, la production annuelle moyenne de 814 millions de $\mathrm{kWh}$ en situation provisoire et de 720 millions de $\mathrm{kWh}$ en situation définitive.

\section{II. - DESCRIPTION DES OUVRAGES}

\section{A) Génie civil}

L'usine de Vogelgrun comprend quatre groupes Kaplan, constituant chacun un bloc indépendant sur un radier général très faiblement armé, et un déchargeur formant un cinquième bloc au centre de l'usine; des locaux administratifs lui sont en outre adjoints sur la rive gauche (fig. $n^{\circ} 1$ ). Ces cinc blocs sont séparés les uns des autres par des joints de dilatation simplement badigeonnés au goudron et dont l'étanchéité est assurée à l'amont et à l'aval par une garniture waterstop type A.

Les bâches spirales, en béton armé, ne supportent que partiellement les étages supérieurs; en effet, les aubes directrices fixes, étant mises en place lors de l'exécution des premiers bétons et non après achèvement du gros ouvre, encaissent une grande partie du poids de ces étages pour le transmettre sur les fondations (fig. $n^{\circ} 4$ ). Ce mode de construction permet d'alléger le ferraillage du plafond des bâches, mais demande en contrepartie un soin particulier dans la mise en place des bétons pour éviter tout déréglage des aubes fixes.

Deux grilles de $46,45 \mathrm{~m} \times 15,70 \mathrm{~m}$ chacune et situées de part et d'autre du déchargeur, l'une au droit des groupes I et II et l'autre au droit des groupes III et JV, protègent les entrées des bâches spirales. Elles sont composées de barreaux en fer plat entretoisés de $14 \mathrm{~mm}$ d'ćpaisseur, $140 \mathrm{~mm}$ de largeur et écartés les uns des autres de $118 \mathrm{~mm}$.
Pour assurer la continuité du débit du canal et ne pas perturber la navigation en cas de baisse de charge ou d'arrêt des groupes, un déchargeur a été prévu (fig. n"1 et 3). Il comprend deux passes rectilignes, chacune étant divisée à l'amont en deux pertuis. Chaque pertuis, de $4,10 \mathrm{~m}$ de large et de $5,60 \mathrm{~m}$ de haut, est capable d'évacuer normalement $300 \mathrm{~m}^{3} / \mathrm{s}$ c'est-à-dire le débit correspondant à celui de chaque turbine, et $250 \mathrm{~m}^{3} / \mathrm{s}$ environ en cas de très fortes crues du Rhin. La dissipation de l'énergie de l'eau évacuée $(190000 \mathrm{ch})$ est assurée par un ressaut hydraulique contrôlé et stabilisé à l'intérieur de chaque passe par un obstacle constitué d'une grille de harreaux de $0,30 \mathrm{~m}$ de diamètre. En outre, pour éviter tout affouillement possible du fond du canal de fuite non revêtu, on a disposć sur une centaine de mètres à l'aval un lit d'enrochements de dimensions décroissantes jusqu'à la granulométrie naturelle du fond du canal. Enfin la concentration du déchargeur au centre de l'usine empêche la dégradation des berges revêtues du canal de fuite.

\section{B) Electro-mécanique}

\section{a) Groupes Générateurs :}

L'usine de Vogelgrun est écquipée, comme celles d'Ottmarsheim et de Fessenheim, de quatre groupes générateurs. Son exploitation est prévue 

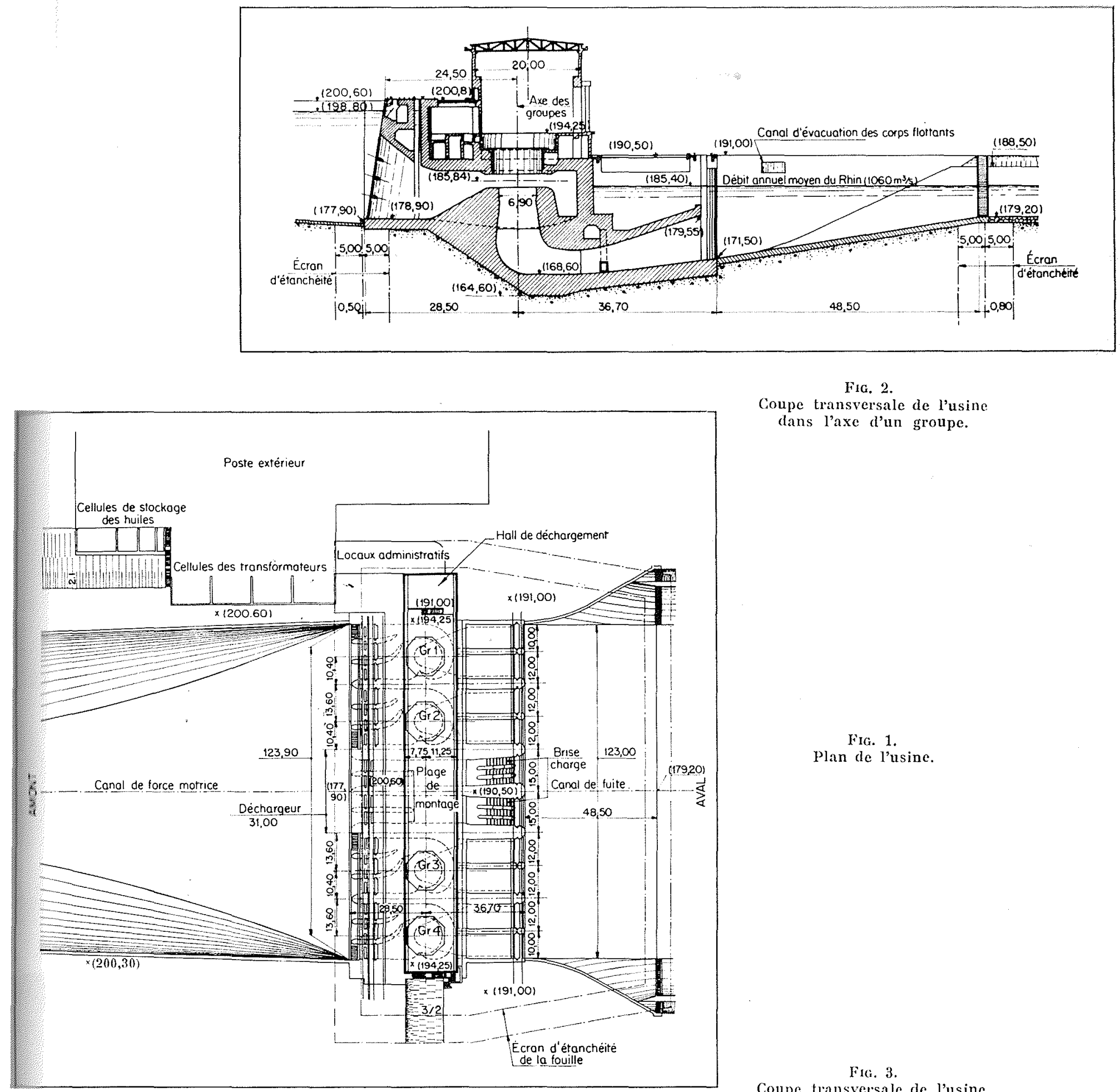

Fic, 2.

Coupe transversale de l'usine dans l'axe d'un groupe.

FIG. 1.

Plan de l'usine.

Fig. 3.

Coupe transversale de l'usine dans une passe du déchargeur.

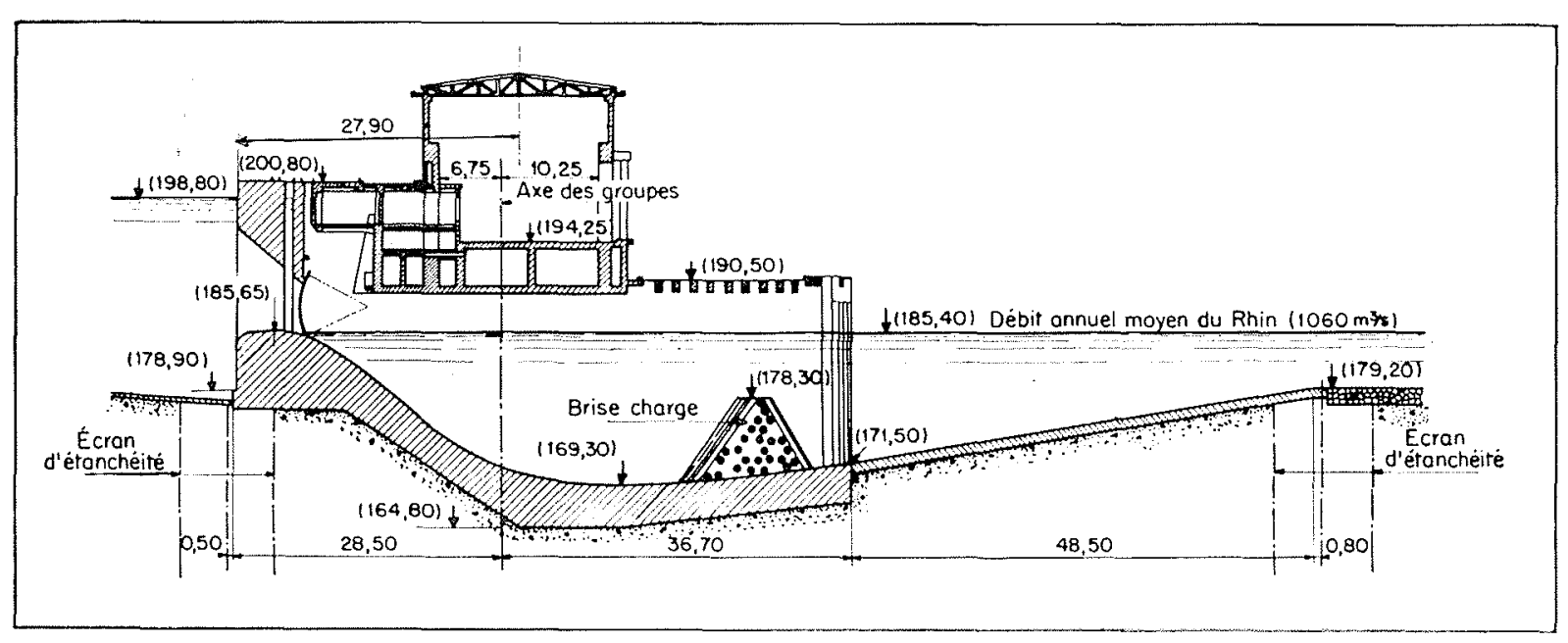




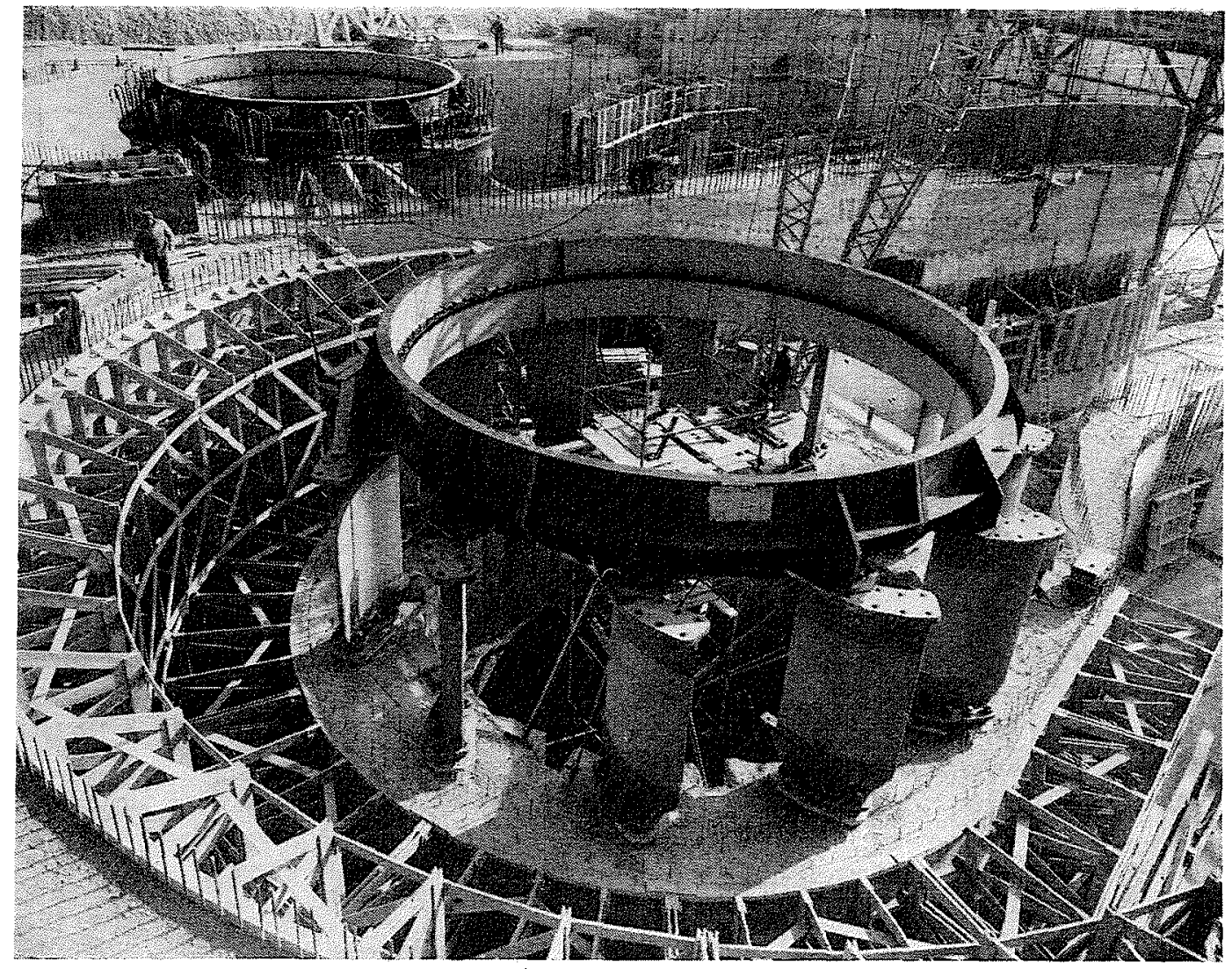

Firi. 4.

Mise en place des aubes directrices fixes et de

la couronne supérieure d'entretoises de ces aubes.

pour le débit concédé de $1080 \mathrm{~m}^{3} / \mathrm{s}$; cependant, pour donner suffisamment d'élasticité aux programmes d'entretien des groupes en raison de leur grand nombre d'heures d'utilisation (7 000 à 8000 heures effectives par an à charge variable), le débit maximum de chaque turbine a été porté à $290 \mathrm{~m}^{3} / \mathrm{s}$, correspondant à un débit total d'équipement de $1160 \mathrm{~m}^{3} / \mathrm{s}$.

Les turbines sont du type Kaplan; elles ont une puissance maximum de $48000 \mathrm{ch}$ correspondant à $290 \mathrm{~m}^{3} / \mathrm{s}$ sous $13,50 \mathrm{~m}$ et une vitesse de rotation de $83 \mathrm{tr} / \mathrm{mn}$. La bâche spirale comprend trois pertuis d'entrée el l'aspirateur deux passes. L'avant-distributeur fixe est constitué de douze aubes directrices en acier moulé. La roue motrice $(125 \mathrm{t})$ de $6,90 \mathrm{~m}$ de diametre comporte quatre pales orientables de $7 \mathrm{t}$ chacune (fig. $n^{\circ} 5$ ).

Les alternateurs sont triphasés et du type «parapluie». Chacun, accouplé directement à la turbine, a une puissance de $39000 \mathrm{kVA}$ sous $10,3 \mathrm{kV}$. Ils sont montés et bobinés sur le chantier, la carcasse du stator étant amenée en quatre éléments. Le stator (123t) a un diamètre extérieur de $11,10 \mathrm{~m}$. Le rolor $(2131)$ a un dia-

Fia. 5. -- Houc de turbine. mètre de $9,57 \mathrm{~m}$ et présente un $\mathrm{PD}^{2}$ de $11500 \mathrm{t} . \mathrm{m}^{2}$. L'arbre principal du groupe $(26 \mathrm{t})$, constitué d'une seule pièce, a une longueur de $6,90 \mathrm{~m}$ et un diametre de $0,85 \mathrm{~m}$.

Le pivot du groupe, situé immédiatement sous

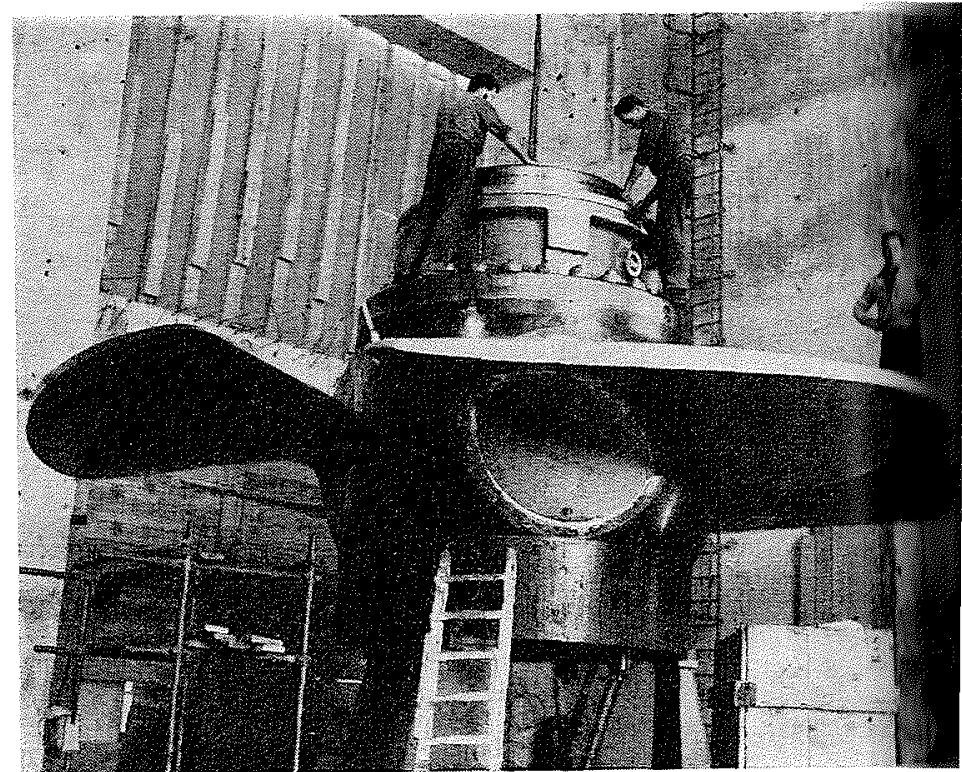


l'alternateur, prend appui par l'intermédiaire d'une chaise sur le couvercle de la turbine solidaire des aubes directrices fixes. Les efforts dus au poids des parties tournantes du groupe (398 $t$ ) et à la poussée hydraulique $(515 t)$ sont done reportés sur ces aubes.

Pour le montage, deux ponls roulants de $130 \mathrm{t}$ chacun permettent l'amenée du matériel nécessaire du hall de déchargement à la plage principale de montage située au centre de l'usine au-dessus du déchargeur (fig. $n^{\circ} 1$ ).

\section{b) Vanves eT BataRdeaUX :}

A l'amont, au droit de l'entrée de chaque bâche spirale, sont installées à demeure trois vannes-wagons de $5,60 \mathrm{~m} \times 10,40 \mathrm{~m}$, composées chacune de quatre éléments articulés et qui peuvent jouer un double rôle : un rôle de batardeaux en cas d'entretien ou de réparation du groupe, un rôle de vannes de sécurité en cas d'emballement de la turbine. Dans ce denier cas, ces vannes descendent en trois minutes à l'aide d'un treuil mis en marche soit automatiquement par la turbine lorsque la vitesse de celle-ci dépasse une certaine valeur, soit manuellement.

A l'aval, la sortie des aspirateurs peut être obturée par de simples batardeaux de $9 \mathrm{~m} \times$ $8,40 \mathrm{~m}$ stockés en rive droite et communs aux deux usines de Fessenheim et de Vogelgrun.

Les quatre vannes-secteurs obturant les pertuis du déchargeur ont chacune $6,35 \mathrm{~m}$ de longueur développée au bordé et $4,10 \mathrm{~m}$ de largeur. Elles peuvent s'ouvrir en 6 secondes soit automatiquement par asservissement à la turbine correspondante, soit manuellement par boutonpoussoir.

\section{c) CrRcuits de puissance:}

Chaque alternateur débite directement sur un transformateur donné (schéma bloc) lui-même relié au réseau général d'interconnexion ì $220 \mathrm{kV}$ ou au réseau régional de $150 \mathrm{kV}$ par l'intermédiaire d'un disjoncteur haute tension.

Les liaisons entre alternateurs et transformateurs sont réalisées par des barres en aluminium enfermées dans des gaines cylindriques.

Les transformateurs triphasés sont du type extérieur à refroidissement par circulation naturelle de l'huile dans des radiateurs à ventilation forcée. Ils offrent la particularité d'avoir un enroulement secondaire permettant de sortir la pleine puissance du groupe, soit à la tension de $150 \mathrm{kV}$, soit à la tension de $220 \mathrm{kV}$.

\section{III. - CONDUITE DES TRAVAUX}

\section{A) Travaux préparatoires.}

Comme à Fessenheim, en raison de l'implantation de l'usine dans les anciennes alluvions perméables du Rhin dont la hauteur atteint ici près de $200 \mathrm{~m}$, nous avons procédé au préalable à la constitution d'une cuvette étanche à l'intérieur de laquelle l'usine fut ensuite fondée.

Cette cuvette, de $175 \mathrm{~m}$ de longueur maximum, $115 \mathrm{~m}$ de largeur et $42,50 \mathrm{~m}$ de profondeur maximum, comprend une enceinte verticale de $14900 \mathrm{~m}^{2}$ de surface totale et un fond de $16500 \mathrm{~m}^{2}$ de surface et $5 \mathrm{~m}$ d'épaisseur (fig. $\mathrm{n}^{\circ} 6,7$ et 8 ). L'enceinte verticale a été constituée à l'aide de trois lignes parallèles de forages distantes de $2 \mathrm{~m}$ l'une de l'autre, le fond à l'aide d'un quadrillage dont la maille avait $10 \mathrm{~m}$ d'écartement.

Au total, $40500 \mathrm{t}$ ont été injectées, dont $34600 \mathrm{t}$ d'argile, $3100 \mathrm{t}$ de ciment et $2.800 \mathrm{t}$ de produits chimiques (aluminate et silicate de soude). Les longueurs de forages ont été de $22500 \mathrm{ml}$, dont $16250 \mathrm{ml}$ pour les écrans verlicaux et $6250 \mathrm{ml}$ pour le fond.

La cuvette a limité les arrivées d'eau à unc soixantaine de litres par seconde alors que sans étanchement elles auraient bien dépassé $12000 \mathrm{l} / \mathrm{s}$ environ d'apris les estimations.

Par suite de la nature alluvionnaire du terrain et de la présence du matelas d'argile constituant le fond de la cuvette étanche sous les fondations de l'usine, on pourrait craindre des tassements inégaux du sol. Des tassements, certes, se produiront, mais l'expérience de l'usine de Fessenheim, construite dans des conditions pratiquement identiques, a montré qu'ils restent dans des limites acceptables ( $2 \mathrm{~cm}$ pendant la construction, $1 \mathrm{~cm}$ pendant la mise en eau). Notons que le calcul indique que la contrainte maximum sur le sol est pour les deux usines de l'ordre de $3 \mathrm{~kg} / \mathrm{cm}^{2}$.

\section{B) Travaux principaux}

$630.000 \mathrm{~m}^{3}$ de déblais ont été exécutés, $178000 \mathrm{~m}^{3}$ de béton, $3500 \mathrm{t}$ d'acier, $110000 \mathrm{~m}^{2}$ de coffrages ont été ou seront mis en œuvre à l'usine, les quantités correspondantes pour l'ensemble de l'aménagement étant de 17 millions de $\mathrm{m}^{3}, 600000 \mathrm{~m}^{3}, 6200 \mathrm{t}, 240000 \mathrm{~m}^{2}$.

Comple tenu de la nature des différentes parties de l'ourrage, il est fait un tres large cmploi $(94 \%)$ de ciments a fort pourcentage de laitier. 


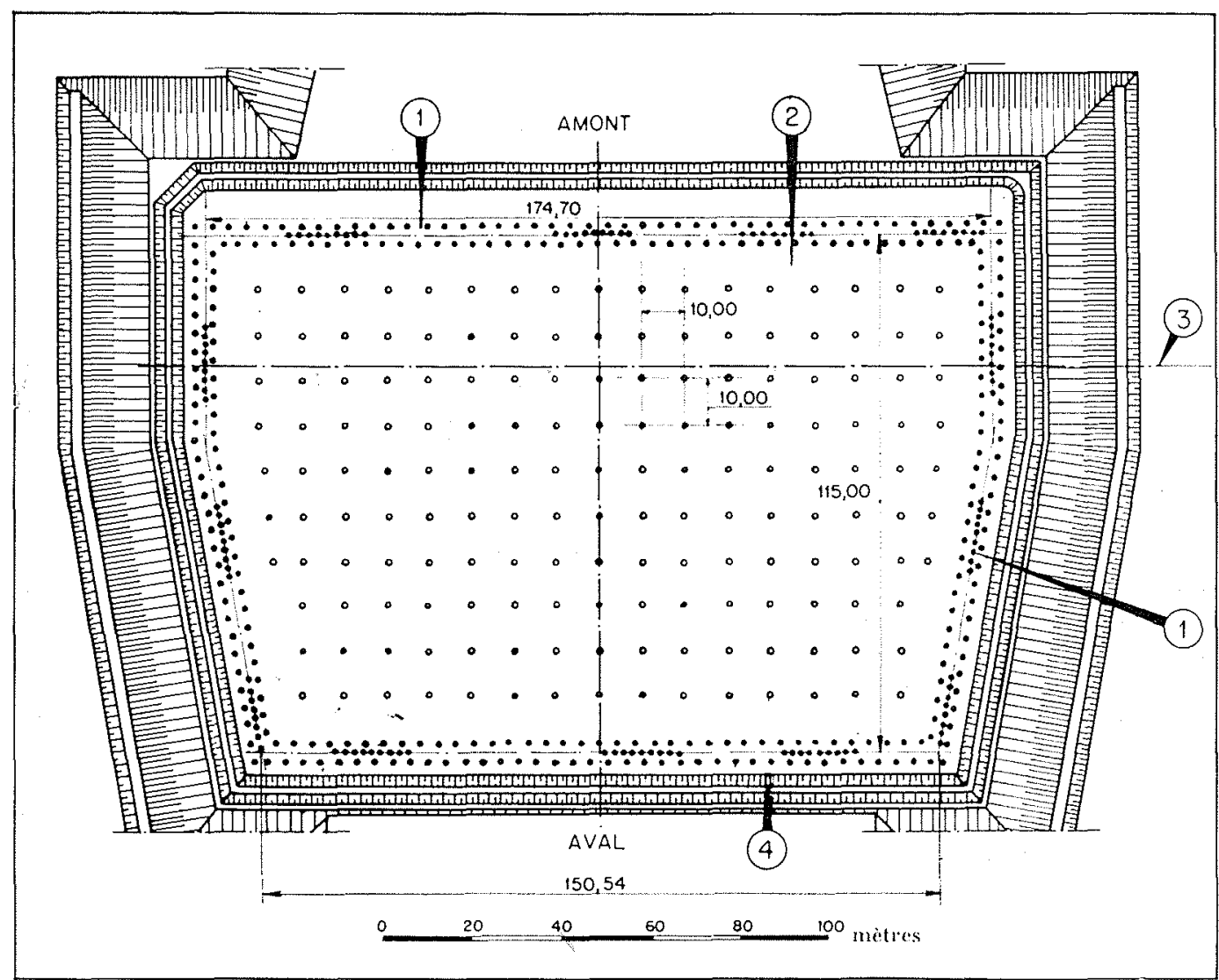

Fic. 6.

Plan.

Cuvette étanche

1. Enceinte verticale.

2. Fond étanche.

3. Axe des groupes.

4. Canal collecteur du rabattement de la nappe à la cote 178,50 .

5. Niveau normal de la nappe.

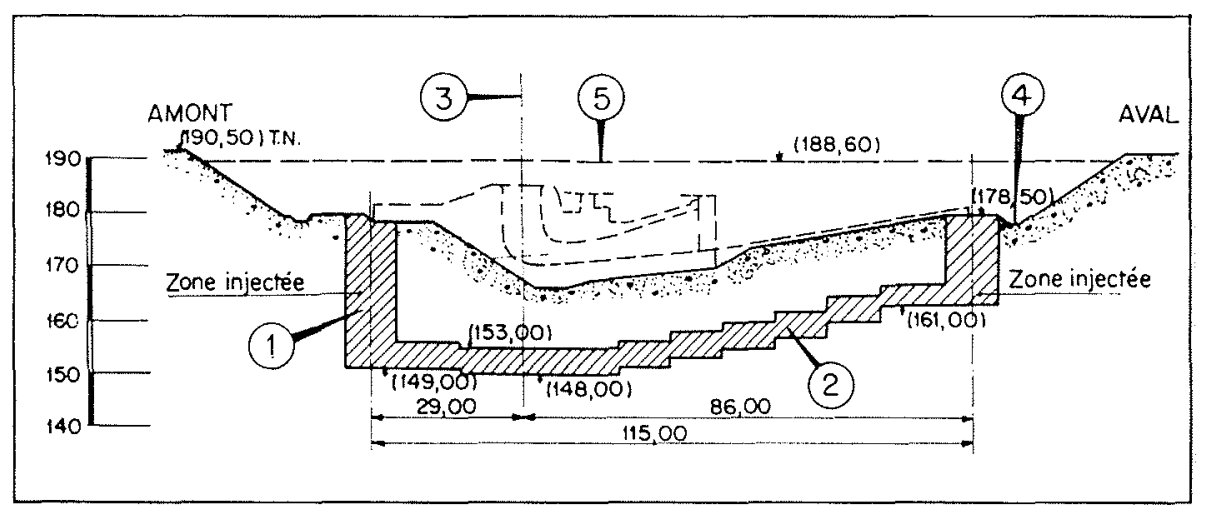

F1G. 7.

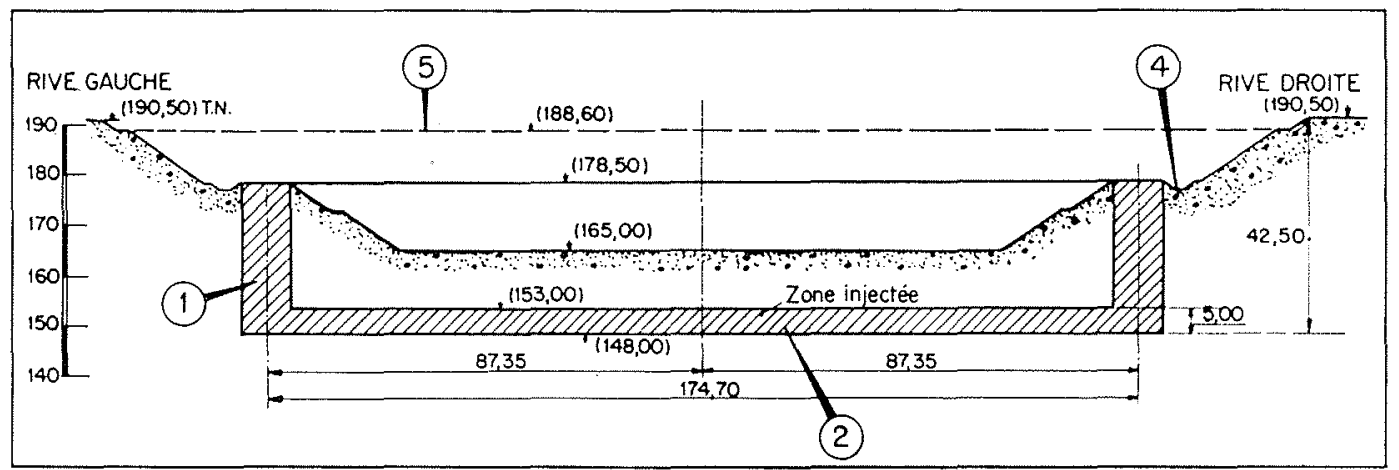

Fic. 8 .

Coupe transversale. 
Fri. 9.

Fouille de l'usine

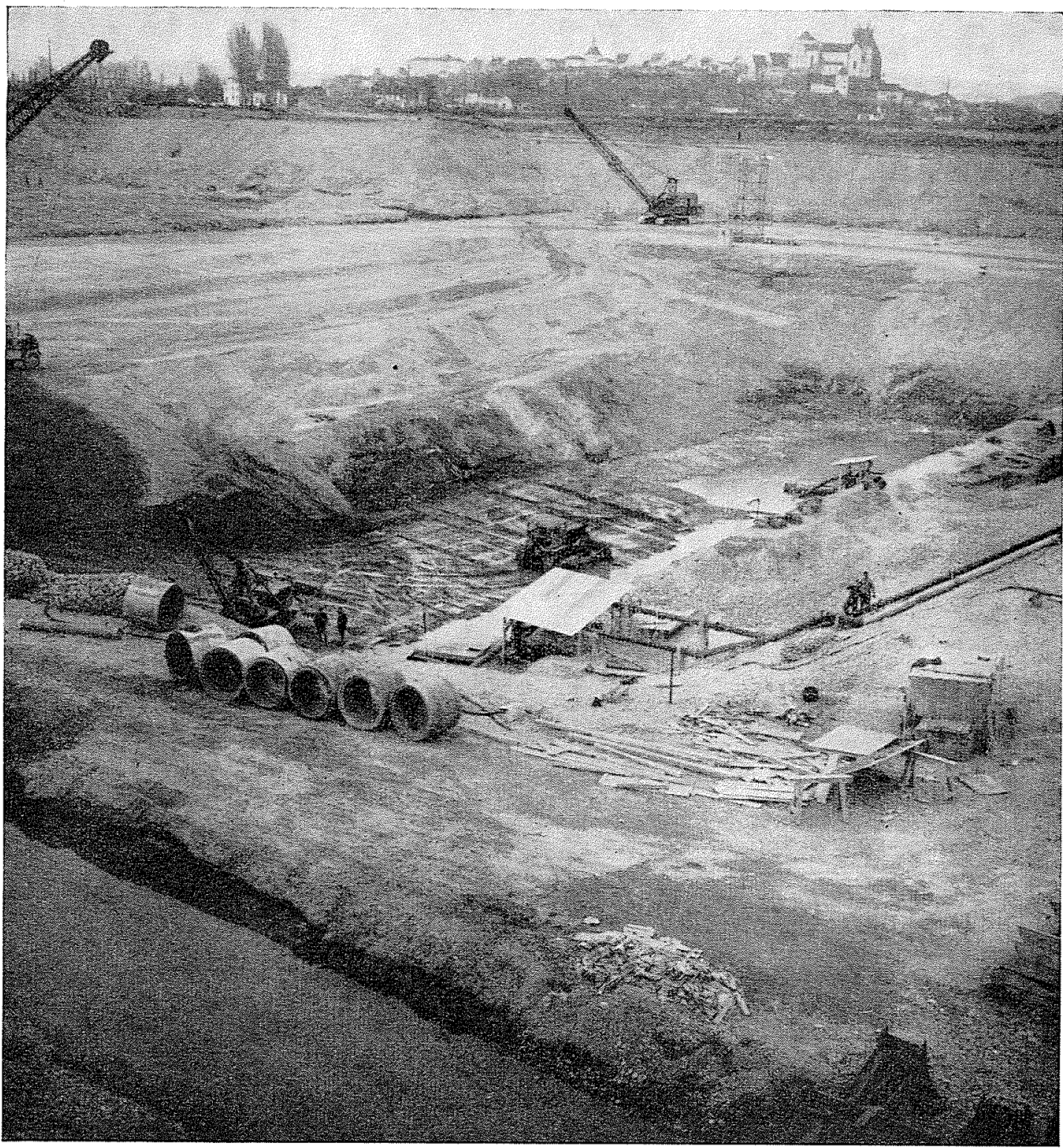

Les agrégats nécessaires à la confection des bétons de l'ensemble de l'aménagement sont tirés des déblais sablo-graveleux du canal de fuite.

Une économie sensible est réalisée sur le poids des ferraillages grâce à la substitution d'une grande partie des aciers doux par des aciers mi-durs crênelés que nous faisons travailler à des taux pouvant atteindre $21 \mathrm{~kg} / \mathrm{mm}^{2}$ au lieu de $14 \mathrm{~kg} / \mathrm{mm}^{2}$.

L'alimentation en énergie électrique pour les besoins de l'aménagement doit être assurée en permanence pour éviter un arrêt des pompages qui entrainerait l'inondation des fouilles; elle est réalisée par une ligne à $150000 \mathrm{~V}$ doublée d'une ligne de secours à $63000 \mathrm{~V}$. La puissance appelée pour les besoins de 'l'ensemble de l'aménagement s'est élevée à $12000 \mathrm{kVA}$, dont $1200 \mathrm{kVA}$ pour les besoins de l'usine.

Les anciennes cités, qui avaient été construites pour les aménagèments précédents, sont utilisées pour loger la majeure partie de la main d'œuvre de Vogelgrun (900 ménages et 360 célibataires pour l'ensemble de l'aménagement, dont 190 et 80 pour l'usine). Le reste de l'effectif (un millie environ pour l'ensemble de l'aménagement) habite la région.

Compte tenu de l'avancement des travaux, nous pensons pouvoir mettre les quatre groupes en service au cours de l'année $\mathbf{1 9 5 9 .}$ 


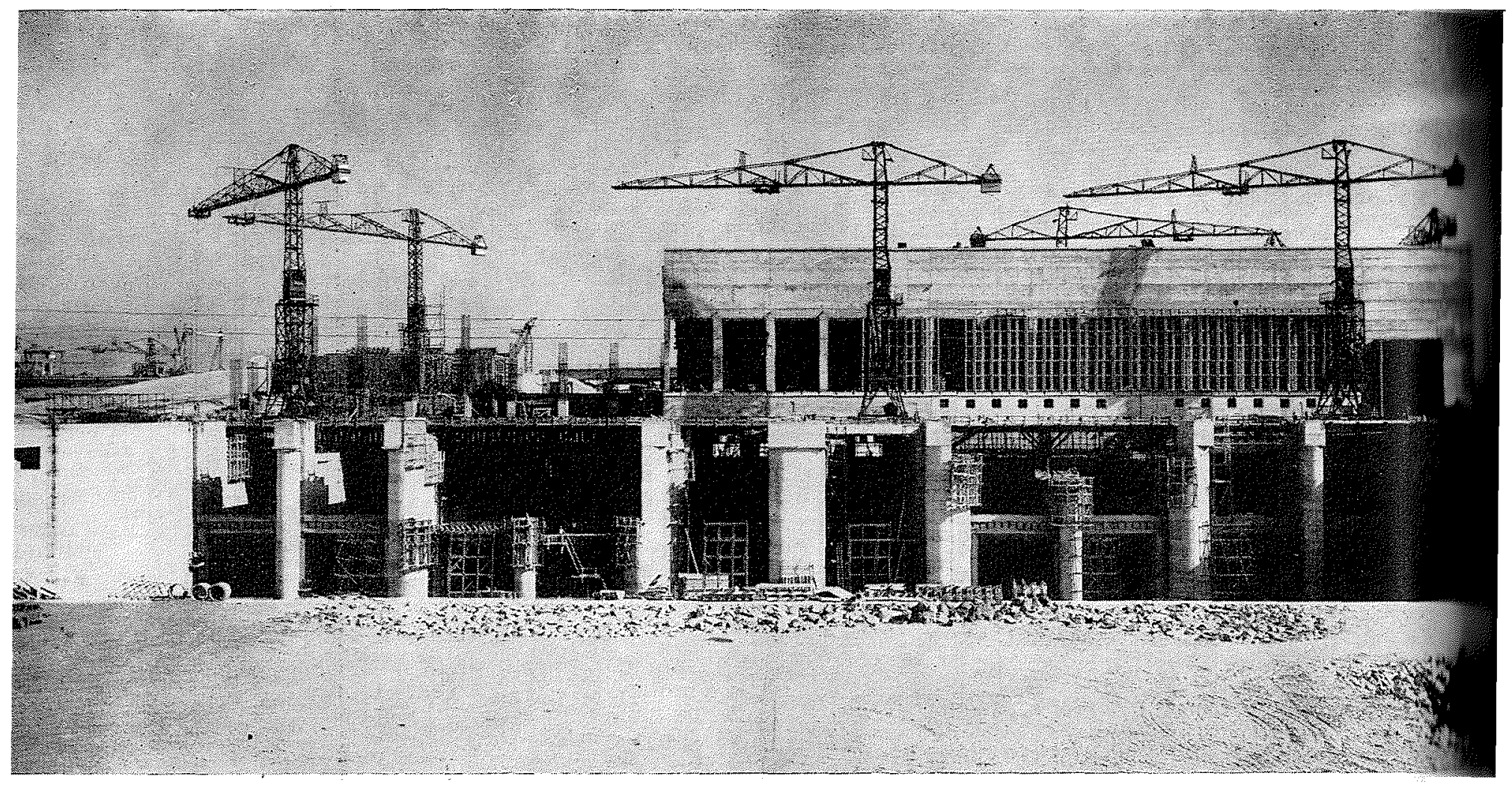

Fig. 10. - Usine, vue de l'aval.

En résumé, l'usine de Vogelgrun, quatrième des huit centrales prévues dans l'aménagement hydro-électrique du Rhin, présente de grandes analogies avec les chutes amont, notamment par son canal d'anenée qui fait suite directement au canal de fuite de Fessenheim, mais elle s'en distingue par le caractère définitif de sa res- titution au Rhin. Comme ce lype de restitution sera également adopté dans les quatre chutes à construire à l'aval, l'usine de Vogelgrun. réalise donc bien la transition entre les deux chaines d'usines amont et aval; c'est la raison qui nous l'a fait traiter à titre d'exemple dans le présent article. 